\title{
INTEGRATION OF CNTS THIN FILM FOR SENSING AND ACTUATING MICRO STRUCTURES
}

\author{
Dau Thanh Van ${ }^{1}$, Bui Thanh Tung ${ }^{2}$, Dao Viet Dzung ${ }^{3}$, Susumu Sugiyama ${ }^{4}$ \\ ${ }^{1}$ Health and Crop Science Laboratory, Sumitomo Chemical Ltd., Hyogo, Japan \\ ${ }^{2}$ National Institute of Advanced Industrial Science and Technology (AIST), Japan \\ ${ }^{3}$ Griffith School of Engineering, Griffith University, Gold Coast Campus, Australia \\ ${ }^{4}$ Graduated School of Science and Engineering, Ritsumeikan University, Shiga, Japan
}

\begin{abstract}
This paper reports the top-down fabrication of CNTs thin film on MEMS structure to develop sensing and actuating micro structures. In particular, this paper review the integration of CNTs film in application of silicon micromirror based on angular vertical comb actuator, development of microstructures with piezoresistive effect and Seebeck effect.
\end{abstract}

Keyword: CNTs thin film, sensor, actuator, Seebeck effect, piezoresistive effect, micromirror.

\section{INTRODUCTION}

Research on carbon nanotubes (CNTs) for sensing and manipulation application is a very promising direction for nanoscale devices due to CNTs excellent electrical and mechanical properties. CNTs possess a tensile strength larger than any other known materials [1]. CNTs have been used in various applications for tunable electrometrical oscillators [2], DNA detection [3], non-volatile memory [4], sensors [5], actuator [6], field-emitting flat panel displays [7] and also nanotube radio [8].

The integration of CNTs in microelectromechanical systems (MEMS) is usually relies either on bottomup (in-situ growth) or top-down (postgrowth) methods. The bottom-up technique utilizes catalytic particles directly patterned onto a substrate to control the position of CNTs, while the top-down method focuses on manipulation of the growth CNTs into the desired positions. Although bottom-up methods have particularly advantages in the area of field-effect transistor, the poor compatibility with MEMS technology is the main obstacle for MEMS integration and functional devices.

In order to accurately deposit the nanotube at the desired location, several topdown methods have been reported. Based on the principle that synthesized SWNTs were preferentially attracted to the $\left(-\mathrm{NH}_{2}\right)$ functionalized surface, Liu et al [9] reported on the surface functionalization technique. This technique can be useful for positioning nanotube but has very poor directional control as the nanotubes often loop around the patterns. Several applications of this method to position nanotubes in between pairs of electrodes have been reported and only limited rate of reliable electrical contact of the device can 
be assured [10]. Huang et al reported on fluidic manipulation, utilizing flow assembly on a functionalized substrate. With a number of control techniques, this method can achieve parallel array of nanotubes and even cross-structure by single or by alternating the flow in orthogonal directions [11]. Another rather fast self-assembly method is dielectrophoretic (DEP) manipulation in which CNTs was trapped and aligned by either or both AC/DC fields. This is the most promising method due to its precise manipulation in non-contact manner, although it is vital to ensure the length of nanotube and to have appropriate electrode size, geometry as well as applied voltage [12-14]. The other top-down technique reported in literatures is nanorobotic manipulations, which directly pick the CNTs by atomic force microscope (AFM) or transmission electron microscope (TEM) and place them at the desired place $[15,16]$ or just use them after random deposition on a substrate without further alignment [17]. The system can operate with sub-nanometer positioning resolution and the nanotubes can be bended, twisted, slided, rolled and broken [18-20]. Obviously, this method is suitable for investigating fundamental properties rather than for large-scale fabrication.

CNTs should have well controlled properties, orientation and be easily integrated to the system in order to be effectively applied to MEMS devices. Recent approach has been reported by Y. Hayamizu et al [21]. This high efficient process was known as "super growth" [22]. The "super growth" CNTs have high purity, and millimeter-scale length. Because of large-scale of the CNTs, conventional MEMS technique can be used to integrate CNTs into microstructures. This process allows complex CNTs component as a fundamental shapeengineerable unit for integrated device system and, therefore, potentially opens a way for low-cost smart CNTs based MEMS devices.

Utilizing the above techniques, this paper introduced some achievements of CNT thin films in fabrication of micro sensing and actuating structures, typically electrostatic micromirror structure demonstrating Seebeck effect and piezoresistive effect by combination of "super growth" and micromachining process.

\section{INTEGRATED CNTS THIN FILM FOR MEMS MECHANICAL SENSORS}

\subsection{Piezoresistive coefficients of aligned single wall carbon nanotube (SWNT) forest film}

One of the main interests of the CNTs properties is the piezoresistive effect. The effect has been investigated for the first time by Tombler et al [23]. A gauge factor (the CNTs sensitivity to strain) of 1000, i.e. five times larger than that of single crystal silicon, was reported. Other measurements by Cao et.al [24] and Stampfer et al. [25] showed the gauge factor up to 3000 for pre-strained individual SWNTs. For practical application, Zhao et al [26] has shown the potentials of SWNT as strain sensor by embedding CNTs in a polymer to measure the stress applied into test specimen. Further study was presented by $\mathrm{Li}$ et al [27] using thin film of nanotubes as strain sensor. The film was made of randomly orientated bundles of SWNTs and was attached to a rubber strip using epoxy. By using DEP method, Sickert et al [28] has presented a macro scale strain sensing device with

bundle single-walled carbon nanotube. Another concept with multi-walled CNTs film was 
reported recently by Li et al [29] and Song et al [30]. Piezoresistive behavior of CNTs was also applied for pressure sensor [31, 32], force sensor [33]. Although the gauge factor was found to be rather small for CNTs films, impressive tensile strength with the elongation over $50 \%$ [34] allows the material to be applied in large deformation sensing device.

Our approach to measure the piezoresistive effect is to pattern the CNTs elements on a silicon bar with dimensions of $50 \times 5 \times 0.5 \mathrm{~mm}^{3}$ by the special top-down process. The $40 \times 5 \times 0.3 \mu \mathrm{m}^{3} \mathrm{CNT}$ elements were aligned with either longitudinal or transverse directions of the bar and connected by $\mathrm{Cr} / \mathrm{Au}$ interconnection to facilitate four-point probe measurement which eliminates unexpected contact resistance (Fig. 1).
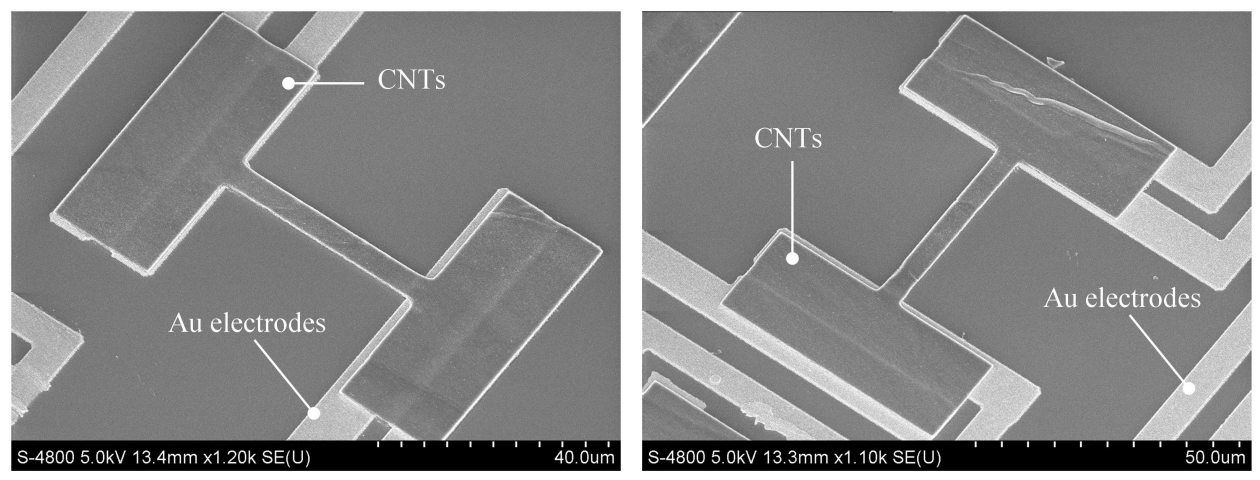

Fig. 1. SEM view of integrated CNTs elements for measurement of piezoresistive effects in longitudinal and tranversal directions

The silicon bar is then subjected to four-point bending test with force indenter applied from its backside (Fig. 2). Since the CNTs is strongly attached to the surface of

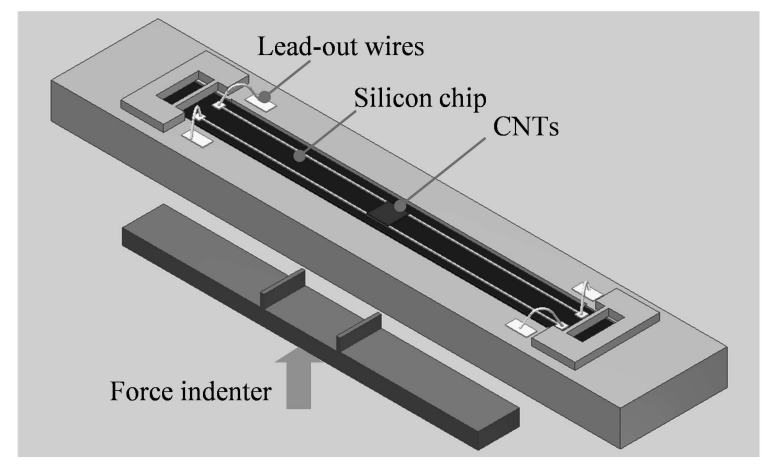

Fig. 2. Schematice view of four-point bending test with CNTs elements

the sample, and the dimension of testing element is ignorable compared with the silicon bar, it is reasonable to assume that the maximum strain in the middle of the bar is 
completely transferred to the CNTs [35]. Strain gauge factor can be expressed as

$$
k=\left(\frac{\Delta R}{R}\right) / \varepsilon
$$

where $\varepsilon$ is the strain in the CNT testing element, $\Delta R$ is the relative change of CNTs resistance.

As CNTs elements were pattern in two directions, the gauge factors were measured simultaneously. The longitudinal and transverse gauge factors, measured when the CNTs is aligned with longitudinal stress direction, are $k_{L}=3.75$ and $k_{T}=0.67$.

With the Young's modulus of CNTs film is 9.7 GPa [22], the piezoresistive coefficients calculated by $(\Delta R / R) / \sigma(\sigma$ is stress $)$ are $\pi_{L}=38.6 \mathrm{e}^{-5} \mathrm{MPa}^{-1}$ and $\pi_{T}=6.91 \mathrm{e}^{-5}$ $\mathrm{MPa}^{-1}$ respectively.

\subsection{Integration of SWNTs film to MEMS for micro thermoelectric device}

Recently, application of CNTs as a thermoelectric material has received the interest for energy sensing, harvesting and power generation. Although direct current (dc) voltage can also be generated due to liquid or gas flow over bundle of CNTs, the generated power is only several picowatts and usually not enough for current micro device application. The thermoelectric power (TEP) or the Seebeck coefficient of CNTs can attain $60 \mu \mathrm{V} / \mathrm{K}$ at room temperature [36]. Thermoelectric properties of CNTs have been investigated by micro-fabricated devices [37-39]. The TEP of individual single-walled CNTs, SWNT ropes and multi-walled CNTs have been reported in the range of $1-100 \mu \mathrm{V} / \mathrm{K}$ [37-49]. Fabrication process's conditions such as pressure, catalysts and impurity, doping condition, orientation, oxygen-exposed or degassed have shown strong effect and can dramatically change the value of TEP especially for the case of bundle or film CNTs sample [50-54].
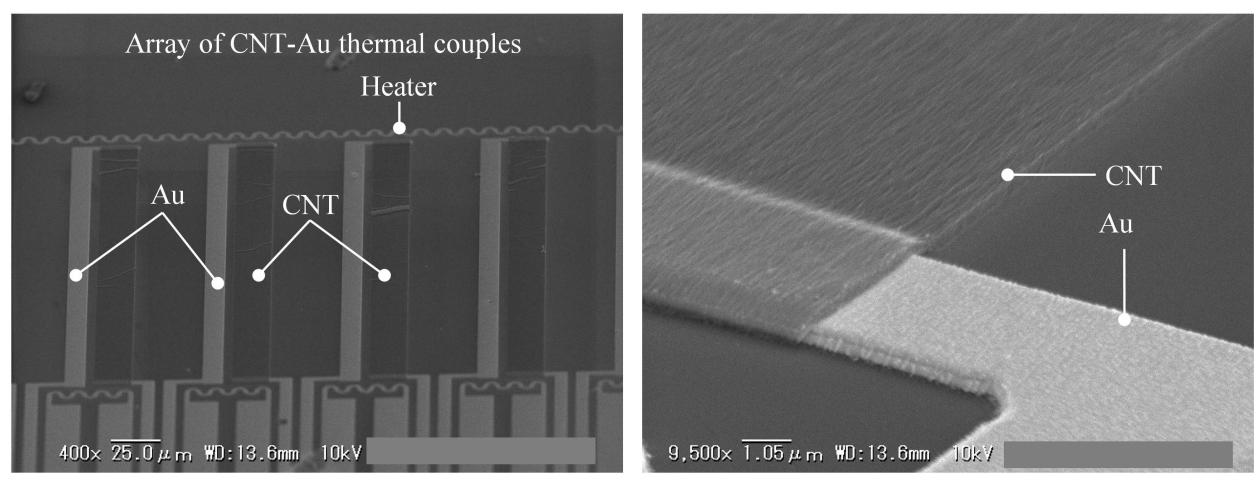

Fig. 3. Fabricated device for Seebeck effect test

Our approach to measure the Seebeck effect of the CNTs film is to utilize the thermocouples made of CNTs-Au as shown in Fig. 3. $0.3 \mu$ m-thick $\mathrm{Cr} / \mathrm{Au}$ temperature sensor, heater, and electrodes were fabricated by photolithography and Au lift-off process on top of silicon substrate covered by $\mathrm{Si}_{3} \mathrm{~N}_{4}$ insulation layer [54]. 
Temperature of the cold-junction was measured by $\mathrm{Au}$ temperature sensor which has dimensions of $150 \times 2 \mu \mathrm{m}^{2}(L \times W)$. Dimensions of CNTs of the thermocouple are $250 \times 20 \mu \mathrm{m}^{2}(L \times W)$. Temperature sensors are located $2 \mu \mathrm{m}$ next to the cold junction of $\mathrm{Au}-\mathrm{CNTs}$ thermocouple, so the measured temperatures can be assumed to be that of the junction with negligible tolerance.

Temperature is recorded by the thermo-resistive effect with measured temperature coefficient of resistance (TCR) of expressed of $2115 \mathrm{ppm} /{ }^{\circ} \mathrm{C}$ in the range of $10^{\circ} \mathrm{C}-70^{\circ} \mathrm{C}$

Seebeck effect is calculated by

$$
V_{s}=\left(S_{C N T}-S_{A u}\right)\left(T_{h o t}-T_{c o l d}\right)
$$

where $S_{C N T}$ and $S_{A u}$ are Seebeck coefficient of CNTs and of Au, respectively, $T_{h o t}$ and $T_{\text {cold }}$ are the temperatures at hot and cold junctions, respectively.

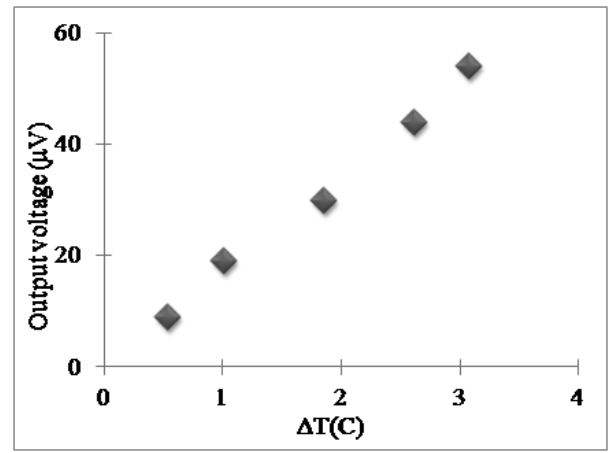

Fig. 4. Output voltage versus temperature differences between two junctions of Au-CNT thermocouple

The output voltage of $54 \mu \mathrm{V}$ was achieved with the different temperature of $3.07^{\circ} \mathrm{C}$, resulting in Seebeck voltage of $19.38 \mu \mathrm{V}$ per Kelvin $(\mu \mathrm{V} / \mathrm{K})$. As the induced voltage is linear with temperature difference in Fig. 4, the Seebeck coefficient is constant.

\section{SCANNING MICROMIRROR WITH CNTs HINGE}

\subsection{System Description}

Electrostatic micromirrors have been widely used in many applications such as displays, barcode scanning, endoscopes optical switches. Among the electrostatic micromirrors, angular vertically comb-driven micromirrors have drawn more attention recently, due to low driving voltage for achieving large angular motion, and less pull-in effect [55-58].

The key technique to develop angular vertical comb actuators is to make the initial tilt angle between the stationary and moveable combs, which is prerequisite to create vertical electrostatic force. Residual stress induced by adding a metallic layer [55] or the surface-tension force resulted from refowing a patterned-photoresist layer [56] had been used for this purpose. Lateral comb drives with mechanical hinges or linkages made of polysilicon [57] or single-crystal silicon [58] have been demonstrated to generate out-ofplane torsional motions. However, the fabrication processes in these studies were still 
complicated and challenging. Moreover, these mirrors required relatively high actuation voltage [55-57] or robust-less [58].

With low Young's modulus and high tensile strength, the CNTs hinge mirrors promise the high flexibility, robustness and low driving voltage. Moreover, CNTs hinges with low resistivity can be used as interconnection wire to apply the driving voltage onto the movable part, extremely convenient compared with another works which required additional metal layer for this function [59].

The movable comb fingers with CNTs hinge tilt out-of-plane with an initial angle at the rest position due to the gravity of the mirror. When a voltage is applied to the vertical comb drive actuator, the tilted and fixed comb fingers will tend to align and thus activating the mirror to rotate. The initial angle of the tilted comb fingers were designed based on the size of CNTs hinge and dimensions of the mirror. The Si mirror has the dimensions of $500 \mu \mathrm{m}$ in length, $155 \mu \mathrm{m}$ in width and $5 \mu \mathrm{m}$ in thickness. The CNT hinge width is $2 \mu \mathrm{m}$ [60].

\subsection{Fabrication process}

Fig. 5 shows the fabrication process of the mirror. CNTs films with dimensions of $500 \times 500 \times 4 \mu \mathrm{m}^{3}(L \times W \times T)$ was synthesized by water-assisted chemical vapor deposition (a) [22]. On a silicon-on-insulator (SOI) wafer (b), a $0.3 \mu \mathrm{m} \mathrm{Cr} / \mathrm{Au}$ electrodes were created

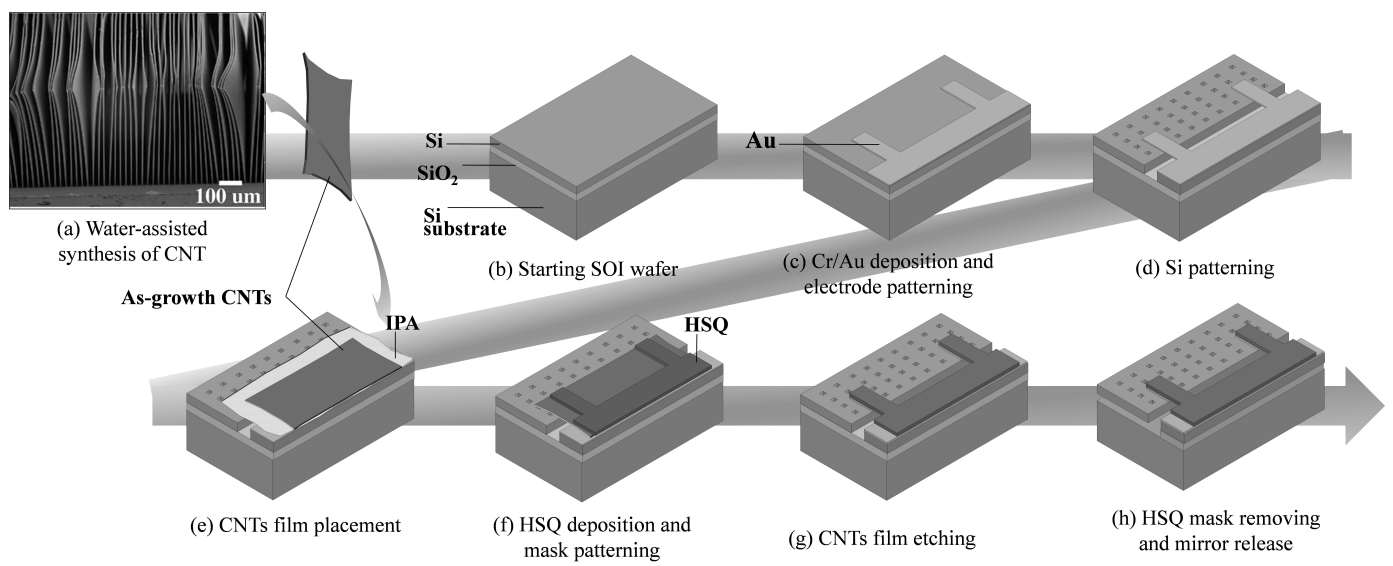

Fig. 5. Integration process of CNTs film into microstructure.

by lift-off process and (c) Next, mirror structure and etching holes are patterned using deep reactive ion etching (D-RIE) (d). CNTs film then is manually placed on the silicon struture in isopropyl alcohol (IPA) solution and naturally dried. Surface tension of solution and strong van der Waals interaction accordantly compress CNTs film into a $0.3 \mu \mathrm{m}$ thick layer and form a strong contact with electrodes (e). Hydrogen silsesquioxane (HSQ) resist was spin-coated and baked at $90^{\circ} \mathrm{C}$ for 10 minutes. HSQ is then patterned by electron beam (EB) lithography and developed by tetramethylammonium hydroxide (TMAH) solution $(2.38 \%)$ (f). Then, CNTs film was etched by reactive ion etching (RIE) to define the designed shape (g). The HSQ mask was finally removed by buffered hydrofluoric acid (HF) 
and mirror is released by HF vapor etching (h). Remarkably, due to parallel process ability, CNTs can be patterned into various desirable array structures and thus has promising application in MEMS devices.

Fig. 6 shows the fabricated CNTs hinge micromirror. Fig. 6 (b) and (c) show the zoom-in of the CNTs hinge and comb fingers, respectively. Because CNTs did not shrink

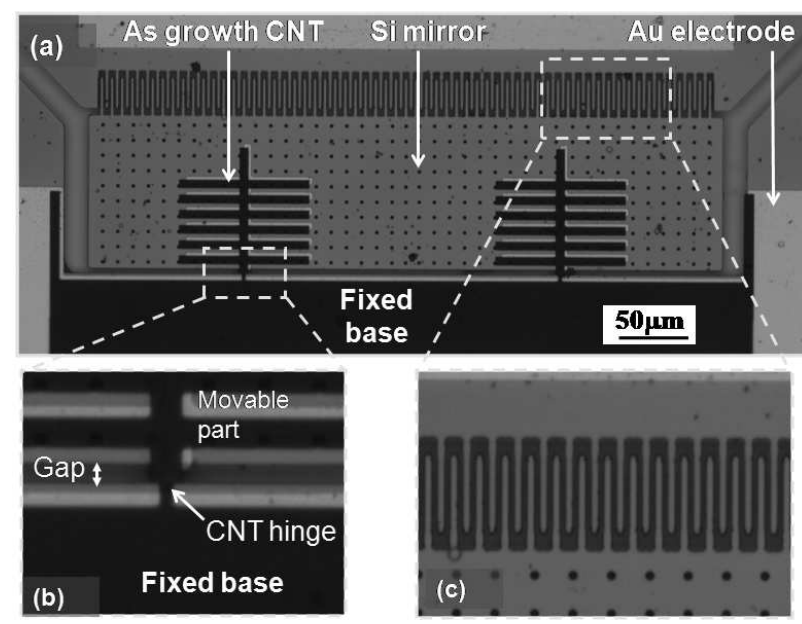

Fig. 6. The fabricated micromirror. (a) Top view of the mirror system. (b) Zoomin of the CNTs hinge. (c) Zoom-in of the comb fingers.

in aligned direction, the film has rather good homogeneity, and the adhesion with the substrate is strong enough to allow successive lithographic processes, include heat treatment, resist spincoating, immersion into liquid and oxygen plasma. After fabrication process, no damage was found on CNTs film elements.

\subsection{Performance characterization}

Fig. 7 gives the measured rotation angle versus the applied voltage. The maximum

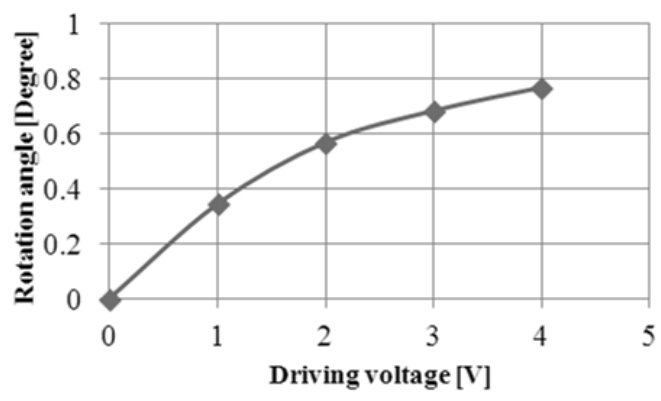

Fig. 7. Rotation of the micromirror versus DC driving voltage.

rotation angle depended on the initial state of the mirror, which is determined by the 
structure parameters. The maximum tilt angle obtained when the rotation angle is saturated, i.e. the mirror parallel with surface plane.

\section{CONCLUSION}

In summary, we have demonstrated the robust fabrication process and its application on fabrication and evaluation of the first silicon MEMS micro mirror supported by CNTs hinges, the micro structures with CNTs as sensing elements by utilizing its piezoresistive effect and Seebeck effect. This process could also advance the further integration of CNTs in MEMS actuator for better and more complex feature.

\section{REFERENCES}

[1] J.-P. Salvetat, G. A. D. Briggs, J.-M. Bonard, R. R. Bacsa, A. J. Kulik, T. Stöckli, N. A. Burnham, and L. Forró, Elastic and Shear Moduli of Single-Walled Carbon Nanotube Ropes, Phys. Rev. Lett., 5(82), (1999), pp. 944-947.

[2] V. Sazonova, Y. Yaish, H. Ustunel, D. Roundy, T. A. Arias, and P. L. McEuen, A tunable carbon nanotube electromechanical oscillator, Nature, 7006(431), (2004), pp. 284-287.

[3] J. Li, H. T. Ng, A. Cassell, W. Fan, H. Chen, Q. Ye, J. Koehne, J. Han, and M. Meyyappan, Carbon Nanotube Nanoelectrode Array for Ultrasensitive DNA Detection, Nano Letters, 5(3), (2003), pp. 597-602.

[4] T. Rueckes, K. Kim, E. Joselevich, G. Y. Tseng, C.-L. Cheung, and C. M. Lieber, Carbon Nanotube-Based Nonvolatile Random Access Memory for Molecular Computing, Science, 5476(289), (2000), pp. 94-97.

[5] S. Ghosh, A. K. Sood, and N. Kumar, Carbon Nanotube Flow Sensors, Science, 5609(299), (2003), pp. 1042-1044.

[6] P. Kim and C. M. Lieber, Nanotube Nanotweezers, Science, 5447(286), (1999), pp. 21482150 .

[7] W. A. de Heer, A. Châtelain, and D. Ugarte, A Carbon Nanotube Field-Emission Electron Source, Science, 5239(270), (1995), pp. 1179-1180.

[8] K. Jensen, J. Weldon, H. Garcia, and A. Zettl, Nanotube Radio, Nano Lett., 11(7), (2007), pp. 3508-3511.

[9] J. Liu, M. J. Casavant, M. Cox, D. A. Walters, P. Boul, W. Lu, A. J. Rimberg, K. A. Smith, D. T. Colbert, and R. E. Smalley, Controlled deposition of individual single-walled carbon nanotubes on chemically functionalized templates, Chemical Physics Letters, 1-2(303), (1999), pp. $125-129$.

[10] J. C. Lewenstein, T. P. Burgin, A. Ribayrol, L. A. Nagahara, and R. K. Tsui, High-Yield Selective Placement of Carbon Nanotubes on Pre-Patterned Electrodes, Nano Lett., 5(2), (2002), pp. 443-446.

[11] Y. Huang, X. Duan, Q. Wei, and C. M. Lieber, Directed Assembly of One-Dimensional Nanostructures into Functional Networks, Science, 5504(291), (2001), pp. 630-633.

[12] W. Salalha and E. Zussman, Investigation of fluidic assembly of nanowires using a droplet inside microchannels, Physics of fluids, 6(17), pp. 063301.1-063301.5.

[13] K. Yamamoto, S. Akita, and Y. Nakayama, Orientation and purification of carbon nanotubes using ac electrophoresis, Journal of Physics D: Applied Physics, 8(31), (1998), pp. L34-L36.

[14] S. Evoy, N. DiLello, V. Deshpande, A. Narayanan, H. Liu, M. Riegelman, B. R. Martin, B. Hailer, J.-C. Bradley, W. Weiss, T. S. Mayer, Y. Gogotsi, H. H. Bau, T. E. Mallouk, and 
S. Raman, Dielectrophoretic assembly and integration of nanowire devices with functional CMOS operating circuitry, Microelectronic Engineering, 1(75), (2004), pp. 31-42.

[15] T. Fukuda, F. Arai, and L. Dong, Assembly of nanodevices with carbon nanotubes through nanorobotic manipulations, Proceedings of the IEEE, 11(91), (2003), pp. 1803 - 1818.

[16] H. W. C. Postma, A. Sellmeijer, and C. Dekker, Manipulation and Imaging of Individual Single-Walled Carbon Nanotubes with an Atomic Force Microscope, Advanced Materials, 17(12), (2000), pp. 1299-1302.

[17] C. Hierold, A. Jungen, C. Stampfer, and T. Helbling, Nano electromechanical sensors based on carbon nanotubes, Sensors and Actuators A: Physical, 1(136), (2007), pp. 51-61.

[18] X. M. H. Huang, R. Caldwell, L. Huang, S. C. Jun, M. Huang, M. Y. Sfeir, S. P. O'Brien, and J. Hone, Controlled Placement of Individual Carbon Nanotubes, Nano Lett., 7(5), (2005), pp. $1515-1518$.

[19] E. W. Wong, P. E. Sheehan, and C. M. Lieber, Nanobeam Mechanics: Elasticity, Strength, and Toughness of Nanorods and Nanotubes, Science, 5334(277), (1997), pp. 1971-1975.

[20] M.-F. Yu, O. Lourie, M. J. Dyer, K. Moloni, T. F. Kelly, and R. S. Ruoff, Strength and Breaking Mechanism of Multiwalled Carbon Nanotubes Under Tensile Load, Science, 5453(287), (2000), pp. 637-640.

[21] Y. Hayamizu, T. Yamada, K. Mizuno, R. C. Davis, D. N. Futaba, M. Yumura, and K. Hata, Integrated three-dimensional microelectromechanical devices from processable carbon nanotube wafers, Nat Nano, 5(3), (2008), pp. 289-294.

[22] K. Hata, D. N. Futaba, K. Mizuno, T. Namai, M. Yumura, and S. Iijima, Water-Assisted Highly Efficient Synthesis of Impurity-Free Single-Walled Carbon Nanotubes, Science, 5700(306), (2004), pp. 1362-1364.

[23] T. W. Tombler, C. Zhou, L. Alexseyev, J. Kong, H. Dai, L. Liu, C. S. Jayanthi, M. Tang, and S.-Y. Wu, Reversible electromechanical characteristics of carbon nanotubes under local-probe manipulation, Nature, 6788(405), (2000), pp. 769-772.

[24] J. Cao, Q. Wang, and H. Dai, Electromechanical Properties of Metallic, Quasimetallic, and Semiconducting Carbon Nanotubes under Stretching, Phys. Rev. Lett., 15(90), (2003), p. 157601.

[25] C. Stampfer, T. Helbling, A. Jungen, and C. Hierold, Piezoresistance of Single-Walled Carbon Nanotubes, Solid-State Sensors, Actuators and Microsystems Conference, 200\%. TRANSDUCERS 200\%. International, (2007), pp. $1565-1568$.

[26] Q. Zhao, J. R. Wood, and H. D. Wagner, Stress fields around defects and fibers in a polymer using carbon nanotubes as sensors, Applied Physics Letters, 12(78), (2001), pp. 1748-1750.

[27] Z. Li, P. Dharap, S. Nagarajaiah, E. V. Barrera, and J. D. Kim, Carbon Nanotube Film Sensors, Advanced Materials, 7(16), (2004), pp. 640-643.

[28] D. Sickert, S. Taeger, I. Kühne, M. Mertig, W. Pompe, and G. Eckstein, Strain sensing with carbon nanotube devices, physica status solidi (b), 13(243), (2006), pp. 3542-3545.

[29] X. Li, C. Levy, and L. Elaadil, Multiwalled carbon nanotube film for strain sensing, Nanotechnology, 4(19), (2008), p. 045501.

[30] X. Song, S. Liu, Z. Gan, Q. Lv, H. Cao, and H. Yan, Controllable fabrication of carbon nanotube-polymer hybrid thin film for strain sensing, Microelectronic Engineering, 11(86), (2009), pp. 2330-2333.

[31] C. K. M. Fung, M. Q. H. Zhang, R. H. M. Chan, and W. J. Li, A PMMA-based micro pressure sensor chip using carbon nanotubes as sensing elements, in 18th IEEE International Conference on Micro Electro Mechanical Systems, 2005. MEMS 2005, (2005), pp. 251 - 254. 
[32] C. Stampfer, T. Helbling, D. Obergfell, B. Schöberle, M. K. Tripp, A. Jungen, S. Roth, V. M. Bright, and C. Hierold, Fabrication of Single-Walled Carbon-Nanotube-Based Pressure Sensors, Nano Lett., 2(6), (2006), pp. 233-237.

[33] C. Stampfer, A. Jungen, and C. Hierold, Fabrication of discrete nanoscaled force sensors based on single-walled carbon nanotubes, IEEE Sensors Journal, 3(6), (2006), pp. 613-617.

[34] M. A. L. Marques, H. E. Troiani, M. Miki-Yoshida, M. Jose-Yacaman, and A. Rubio, On the Breaking of Carbon Nanotubes under Tension, Nano Lett., 4, 5, (2004), pp. 811-815.

[35] V. T. Dau, T. Yamada, D. Viet Dao, B. Thanh Tung, K. Hata, and S. Sugiyama, Integrated CNTs thin film for MEMS mechanical sensors, Microelectronics Journal, 12(41), (2010), pp. 860-864.

[36] A. K. Sood and S. Ghosh, Direct Generation of a Voltage and Current by Gas Flow Over Carbon Nanotubes and Semiconductors, Phys. Rev. Lett., 8(93), (2004), p. 086601.

[37] P. Kim, L. Shi, A. Majumdar, and P. L. McEuen, Thermal transport measurements of individual multiwalled nanotubes, arXiv:cond-mat/0106578, (2001).

[38] L. Shi, D. Li, C. Yu, W. Jang, D. Kim, Z. Yao, P. Kim, and A. Majumdar, Measuring Thermal and Thermoelectric Properties of One-Dimensional Nanostructures Using a Microfabricated Device, Journal of Heat Transfer, 5(125), (2003), p. 881.

[39] J. P. Small, K. M. Perez, and P. Kim, Modulation of thermoelectric power of individual carbon nanotubes, Phys. Rev. Lett., 25(91), (2003), p. 256801.

[40] M. Tian, F. Li, L. Chen, Z. Mao, and Y. Zhang, Thermoelectric power behavior in carbon nanotubule bundles from 4.2 to $300 \mathrm{~K}$, Physical Review B, 3(58), (1998), pp. 1166-1168.

[41] M. C. Llaguno, J. E. Fischer, A. T. Johnson, and J. Hone, Observation of Thermopower Oscillations in the Coulomb Blockade Regime in a Semiconducting Carbon Nanotube, Nano Lett., 1(4), (2004), pp. 45-49.

[42] J. Hone, M. C. Llaguno, N. M. Nemes, A. T. Johnson, J. E. Fischer, D. A. Walters, M. J. Casavant, J. Schmidt, and R. E. Smalley, Electrical and thermal transport properties of magnetically aligned single wall carbon nanotube films, Applied Physics Letters, 5(77), (2000), pp. $666-668$.

[43] J. Vavro, M. C. Llaguno, J. E. Fischer, S. Ramesh, R. K. Saini, L. M. Ericson, V. A. Davis, R. H. Hauge, M. Pasquali, and R. E. Smalley, Thermoelectric Power of p-Doped Single-Wall Carbon Nanotubes and the Role of Phonon Drag, Phys. Rev. Lett., 6(90), (2003), p. 065503.

[44] W. J. Kong, L. Lu, H. W. Zhu, B. Q. Wei, and D. H. Wu, Thermoelectric power of a singlewalled carbon nanotubes strand, Journal of Physics: Condensed Matter, 12(17), (2005), pp. $1923-1928$.

[45] H. E. Romero, G. U. Sumanasekera, G. D. Mahan, and P. C. Eklund, Thermoelectric power of single-walled carbon nanotube films, Phys. Rev. B, 20(65), (2002), p. 205410.

[46] J. Hone, I. Ellwood, M. Muno, A. Mizel, M. Cohen, A. Zettl, A. Rinzler, and R. Smalley, Thermoelectric Power of Single-Walled Carbon Nanotubes, Physical Review Letters, 5(80), (1998), pp. 1042-1045.

[47] N. Kang, L. Lu, W. J. Kong, J. S. Hu, W. Yi, Y. P. Wang, D. L. Zhang, Z. W. Pan, and S. S. Xie, Observation of a Logarithmic Temperature Dependence of Thermoelectric Power on Multiwall Carbon Nanotubes, arXiv:cond-mat/0202065, (2002).

[48] M. Baxendale, K. G. Lim, and G. A. J. Amaratunga, Thermoelectric power of aligned and randomly oriented carbon nanotubes, Phys. Rev. B, 19(61), (2000), pp. 12705-12708.

[49] S. Berber, Y.-K. Kwon, and D. Tománek, Unusually High Thermal Conductivity of Carbon Nanotubes, Phys. Rev. Lett., 20(84), (2000), pp. 4613-4616. 
[50] G. U. Sumanasekera, C. K. W. Adu, S. Fang, and P. C. Eklund, Effects of Gas Adsorption and Collisions on Electrical Transport in Single-Walled Carbon Nanotubes, Phys. Rev. Lett., 5(85), (2000), pp. 1096-1099.

[51] K. Bradley, S.-H. Jhi, P. Collins, J. Hone, M. Cohen, S. Louie, and A. Zettl, Is the Intrinsic Thermoelectric Power of Carbon Nanotubes Positive?, Physical Review Letters, 20(85), (2000), pp. 4361-4364.

[52] P. G. Collins, K. Bradley, M. Ishigami, and A. Zettl, Extreme Oxygen Sensitivity of Electronic Properties of Carbon Nanotubes, Science, 5459(287), (2000), pp. 1801-1804.

[53] I. Kunadian, R. Andrews, M. Pinar Mengüç, and D. Qian, Thermoelectric power generation using doped MWCNTs, Carbon, 3(47), (2009), pp. 589-601.

[54] V. T. Dau, D. V. Dao, T. Yamada, B. T. Tung, K. Hata, and S. Sugiyama, Integration of SWNT film into MEMS for a micro-thermoelectric device, Smart Mater. Struct., 7(19), (2010), p. 075003.

[55] Huikai Xie, Yingtian Pan, and G. K. Fedder, A CMOS-MEMS mirror with curled-hinge comb drives, Microelectromechanical Systems, Journal of, 4(12), (2003), pp. 450-457.

[56] P. R. Patterson, Dooyoung Hah, Hung Nguyen, H. Toshiyoshi, Ru-min Chao, and M. C. Wu, A scanning micromirror with angular comb drive actuation, Micro Electro Mechanical Systems, 2002. The Fifteenth IEEE International Conference on, (2002), pp. 544-547.

[57] Meng-Hsiung Kiang, O. Solgaard, K. Y. Lau, and R. S. Muller, Electrostatic combdriveactuated micromirrors for laser-beam scanning and positioning, Microelectromechanical Systems, Journal of, 1(7), (1998), pp. 27-37.

[58] V. M. Matthew, M. Last, and K. S. J. Pister, Torsional Micromirrors with Lateral Actuators, Transducers'01 - Eurosensors XV conference, Muenchen, (2001), pp. 1298-1301.

[59] J. Chung and W. Hsu, Fabrication of a polymer-based torsional vertical comb drive using a double-side partial exposure method, J. Micromech. Microeng., 3(18), (2008), p. 035014.

[60] B. T. Tung, V. T. Dau, D. V. Dao, T. Yamada, K. Hata, and S. Sugiyama, Fabrication and characterization of silicon micro mirror with CNT hinge, in Micro Electro Mechanical Systems (MEMS), 2011 IEEE 24th International Conference on, (2011), pp. 688 -691.

Received July 31, 2012 